\title{
Neutrino 2-3 Symmetry and Inverted Hierarchy
}

\author{
C.S. Lam \\ Department of Physics and Astronomy, \\ University of British Columbia, \\ Vancouver, BC, Canada V6T $1 Z 1$ \\ and \\ Theory Group, TRIUMF, \\ Vancouver, BC, Canada V6T 2A3 \\ Lam@physics.ubc.ca
}

\begin{abstract}
Neutrino oscillation data indicate the presence of a 2-3 symmetry for the left-handed neutrinos. We show that this symmetry between the second and third generations cannot be extended to include the charged leptons, no matter what basis we use. We point out that if this symmetry is also independently valid for the right-handed neutrinos, then the active neutrino spectrum is inverted, with $m_{3}=0$. This conclusion remains valid even when the left-handed 2-3 symmetry is broken by a non-maximal atmospheric mixing angle $\theta_{23}$, or a non-zero reactor angle $\theta_{13}$. As previously shown by Mohapatra, Nasri, and Yu, such a symmetry also gives rise to interesting consequences on the leptogenesis asymmetry parameter $\epsilon_{1}$.
\end{abstract}




\section{2-3 SYMMETRY FOR LEFT-HANDED LEPTONS}

The leptonic mass Lagrangian can be written symbolically as

$$
\mathcal{L}=-\bar{L}_{e} M_{e} R_{e}-L_{\nu}^{T} M_{\nu} L_{\nu}
$$

where $L_{e}, L_{\nu}$ are the left-handed fields for the charged leptons and neutrinos, and $R_{e}, R_{\nu}$ are the corresponding right-handed fields. $M_{e}$ is the $3 \times 3$ charged lepton mass matrix, and $M_{\nu}$ is the symmetric mass matrix for left-handed Majorana neutrinos. In type-I seesaw, this Majorana mass is related to the Dirac mass $-\bar{L}_{\nu} M_{D} R_{\nu}$ and the right-handed Majorana mass $-R_{\nu}^{T} M_{R} R_{\nu}$ by

$$
M_{\nu}=M_{D}^{T} M_{R}^{-1} M_{D}
$$

In the basis where $M_{e}=\operatorname{diag}\left(m_{e}, m_{\mu}, m_{\tau}\right)$ is diagonal, $M_{\nu}$ can be diagonalized by the unitary MNS mixing matrix [1] $U, M_{\nu}=U^{T} M_{\nu}^{d} U$, with $M_{\nu}^{d}=\operatorname{diag}\left(m_{1}, m_{2}, m_{3}\right)$ being

the (generally complex) neutrino mass parameters. Data are consistent [2] with having the atmospheric mixing angle $\theta_{23}$ maximal, and the reactor angle $\theta_{13}$ zero. In that case, $U$ can be parameterized as

$$
U=\frac{1}{\sqrt{2}}\left(\begin{array}{ccc}
\sqrt{2} c & \sqrt{2} s & 0 \\
-s & c & 1 \\
-s & c & -1
\end{array}\right)
$$

where $s=\sin \theta_{12}, c=\cos \theta_{12}$, and $\theta_{12}$ is the solar mixing angle.

The resulting neutrino mass matrix

$$
M_{\nu}=U^{T} M_{\nu}^{d} U=\left(\begin{array}{lll}
\nu_{11} & \nu_{12} & \nu_{12} \\
\nu_{12} & \nu_{22} & \nu_{23} \\
\nu_{12} & \nu_{23} & \nu_{22}
\end{array}\right),
$$

with

$$
\begin{aligned}
\nu_{11} & =c^{2} m_{1}+s^{2} m_{2} \\
\nu_{12} & =c s\left(m_{2}-m_{1}\right) / \sqrt{2} \\
\nu_{22} & =\frac{1}{2}\left(s^{2} m_{1}+c^{2} m_{2}+m_{3}\right) \\
\nu_{23} & =\frac{1}{2}\left(s^{2} m_{1}+c^{2} m_{2}-m_{3}\right),
\end{aligned}
$$


is invariant under the simultaneous interchanges of the second and third columns, together with the second and third rows. We shall refer to this symmetry as the 2-3 symmetry for left-handed neutrinos.

Conversely, the invariance of $\mathcal{L}$ under a permutation of the second and third generations $L_{\nu}$, via the matrix

$$
P=\left(\begin{array}{lll}
1 & 0 & 0 \\
0 & 0 & 1 \\
0 & 1 & 0
\end{array}\right)
$$

will result in an identity $L_{\nu}^{T} P M_{\nu} P L_{\nu}=L_{\nu}^{T} M_{\nu} L_{\nu}$, or equivalently $P M_{\nu} P=M_{\nu}$. The most general symmetric matrix satisfying this constraint is of the form given by (4). Moreover, a matrix of this form is known to lead to a maximum $\theta_{23}$ and a zero $\theta_{13}[3,4]$.

Since left-handed neutrinos have a 2-3 symmetry, one might wonder whether the lefthanded charged leptons also have the same symmetry. In the basis where the charged leptons are diagonal, clearly they do not, because $m_{\mu} \neq m_{\tau}$. Actually, a 2-3 symmetry cannot be simultaneously valid for the left-handed charged leptons and the left-handed neutrinos, no matter what basis we choose. This can be seen as follows.

If both $L_{\nu}$ and $L_{e}$ are 2-3 symmetric, then $P M_{\nu}=M_{\nu} P$, and $P H_{e}=H_{e} P$, where $H_{e}=M_{e} M_{e}^{\dagger}$. Since they commute, there must be a unitary matrix $V_{\nu}$ that can diagonalize $P$ and $M_{\nu}$ simultaneously, and another unitary matrix $V_{e}$ that can diagonalize $P$ and $H_{e}$ simultaneously. Now the matrix $S$ below diagonalizes $P$,

$$
S^{\dagger} P S=\left(\begin{array}{ccc}
1 & 0 & 0 \\
0 & 1 & 0 \\
0 & 0 & -1
\end{array}\right) \quad \text { for } \quad S=\left(\begin{array}{ccc}
1 & 0 & 0 \\
0 & 1 / \sqrt{2} & 1 / \sqrt{2} \\
0 & 1 / \sqrt{2} & -1 / \sqrt{2}
\end{array}\right) \text {, }
$$

but since $P$ has two degenerate eigenvalues +1 , this diagonalization is not unique. The most general unitary matrix to diagonalize $P$ is of the form $V=S W$, with

$$
W=\left(\begin{array}{cc}
w & 0 \\
0 & e^{i \phi}
\end{array}\right),
$$

and $w$ any $2 \times 2$ unitary matrix. The neutrino mixing matrix $U=V_{e}^{\dagger} V_{\nu}=W_{e}^{\dagger} W_{\nu}$ is therefore block diagonal, implying no mixing of the third-generation neutrino with the other two neutrino generations. This is manifestly false, hence the 2-3 symmetry cannot be simultaneously true for $L_{e}$ and $L_{\nu}$ in any basis. 
We do not understand why the 2-3 symmetry is (at least approximately) true for the left-handed neutrinos but not for the left-handed charged leptons, though there are models that can describe it [4], but data indicate that to be so. From now on we will concentrate solely on the neutrino symmetry.

\section{2-3 SYMMETRY FOR THE RIGHT-HANDED NEUTRINOS}

Given a mass matrix $M_{\nu}$ in (4) and (5), the seesaw formula (2) is insufficient to determine both the Dirac mass matrix $M_{D}$ and the right-handed Majorana mass matrix $M_{R} . M_{R}$, being symmetric, contains 6 complex parameters, but $M_{D}$, not having any symmetry, generally contains 9. Since $M_{D}^{T} M_{R}^{-1} M_{D}$ is symmetric, the seesaw formula contains 6 constraints, thereby leaving 9 complex parameters in $M_{D}$ and $M_{R}$ undetermined. If we want to use neutrino oscillation to probe the dynamics beyond the Standard Model, we need to know those parameters. In any case, some combinations of these parameters are actually required to calculate the asymmetry parameter $\epsilon_{1}$ in leptogenesis.

One might be able to reduce the number of undetermined parameters if the right-handed neutrinos obey some symmetry. Since the left-handed neutrinos exhibit a 2-3 symmetry, we shall assume the right-handed neutrinos to possess a 2-3 symmetry as well. This includes the possibilities of having the right-handed neutrinos possess a 1-2 or a 1-3 symmetry instead, because a relabeling of generations of the right-handed neutrinos will convert those symmetries into a 2-3 symmetry.

The right-handed 2-3 symmetry might be coupled and linked to the left-handed 2-3 symmetry, or it might be an independent symmetry not linked to the left-handed one. Let us look at these two cases separately.

In the first case, $\bar{L}_{\nu} M_{D} R_{\nu}=\bar{L}_{\nu} P M_{D} P R_{\nu}$ and $R_{\nu}^{T} M_{R} R_{\nu}=R_{\nu}^{T} P M_{R} P R_{\nu}$, so the constraints on the mass matrices are $M_{D}=P M_{D} P$, and $M_{R}=P M_{R} P$. The constraint on $M_{R}$ is identical to the constraint on $M_{\nu}$, so $M_{R}$ contains 6 complex parameters. $M_{D}$, not being symmetric, contains 7 complex parameters. The number of undetermined parameters is now reduced from 9 to 7 .

As shown by Mohapatra and Nasri [5], and also by Grimus and Lavoura [6], the leptogenesis asymmetry parameter $\epsilon_{1}$ is then proportional to $\Delta m_{\odot}^{2}$, rather than the more generic $\Delta m_{\text {atm }}^{2}[7]$. 
If the right-handed 2-3 symmetry is independent of the left-handed 2-3 symmetry, then the constraint on $M_{R}$ remains the same, but the constraint on $M_{D}$ becomes stronger. In that case, $\bar{L}_{\nu} M_{D} R_{\nu}=\bar{L}_{\nu} P M_{D} R_{\nu}=\bar{L}_{\nu} M_{D} P R_{\nu}$, hence $M_{D}=P M_{D}=M_{D} P$. In other words, the second and third columns of $M_{D}$ must be identical, and so must be the second and third rows. Thus $M_{D}$ is of the form

$$
M_{D}=\left(\begin{array}{lll}
a & b & b \\
d & c & c \\
d & c & c
\end{array}\right),
$$

which is specified by 4 complex parameters. In this case the number of unknown parameters is further reduced from 7 to 4 .

The conclusion of Ref. [5] and [6] on $\epsilon_{1}$ remains valid, but there are now additional consequences which we shall discuss in the rest of this article.

Since $M_{D}$ has two identical columns, its determinant is zero. Hence $M_{\nu}$ given by (2) also has a zero determinant, and thus a zero eigenvalue. This eigenvalue is $m_{3}$, as can be seen as follows. Compute $S^{T} M_{\nu} S$, where $M_{\nu}$ is the matrix in (4). It yields a block diagonal matrix, with the $13,23,31$, and 32 matrix elements zero, and the 33 matrix element equal to $\nu_{22}-\nu_{23}$. Using (5), this difference is equal to $m_{3}$. Now compute $S^{T} M_{\nu} S$ again, this time using $M_{\nu}$ given by (2). With $M_{R} 2-3$ symmetric and $M_{D}$ given by (9), a straight forward calculation shows that the resulting 33 matrix element is zero. Hence $m_{3}=0$.

With $m_{3}=0$, the magnitudes of the remaining neutrino masses are determined by the atmospheric and solar mass gaps to be

$$
\begin{aligned}
& \left|m_{1}\right|=\sqrt{\Delta m_{\text {atm }}^{2}} \simeq 52 \mathrm{meV} \\
& \left|m_{2}\right|=\sqrt{\Delta m_{\text {atm }}^{2}+\Delta m_{\odot}^{2}} \simeq 53 \mathrm{meV} .
\end{aligned}
$$

The sum $\sum_{i=1}^{2}\left|m_{i}\right|$, just over $0.1 \mathrm{eV}$, is comfortably below the upper bound of $0.47 \mathrm{eV}$ placed by astrophysical data, including Ly $\alpha[8]$. The effective neutrino mass measured in tritium $\beta$-decay is

$$
m_{\nu_{e}}=\sqrt{\sum_{i}\left|U_{e i}^{2} m_{i}^{2}\right|}=\sqrt{c^{2}\left|m_{1}\right|^{2}+s^{2}\left|m_{2}\right|^{2}},
$$

which lies between $\left|m_{1}\right|$ and $\left|m_{2}\right|$. This is to be compared with the $2.2 \mathrm{eV}$ upper bound from experiments [9]. Unfortunately, this number is too low to be reached by KATRIN for 
verification. Finally, the effective mass for the neutrinoless double $\beta$-decay is

$$
\begin{aligned}
m_{e e} & =\left|\sum_{i=1}^{3} U_{e i}^{2} m_{i}\right|=\left|c^{2} m_{1}+s^{2} m_{2}\right|=\sqrt{\left(c^{2}\left|m_{1}\right|+s^{2}\left|m_{2}\right|\right)^{2}-\left|m_{1} m_{2}\right| \sin ^{2} 2 \theta_{\odot} \sin ^{2} \frac{\phi_{12}}{2}} \\
& \simeq\left|m_{1}\right| \sqrt{1-0.84 \sin ^{2} \frac{\phi_{12}}{2}}
\end{aligned}
$$

where $\phi_{12}$ is the relative Majorana phase angle between $m_{1}$ and $m_{2}$. The current upper bound from experiment is about $0.3 \mathrm{eV}$, with a factor of 3 uncertainty from the nuclear matrix elements [10].

\section{BREAKING THE LEFT-HANDED 2-3 SYMMETRY}

If $\theta_{23}$ is not exactly maximal, or $\theta_{13} \neq 0$, then the 2-3 symmetry for the left-handed neutrino is broken. Nevertheless, present experiments indicate that the breaking has to be small.

We can quantify the breaking in the following way. Under a 2-3 permutation of the lefthand neutrino field, $L_{\nu} \rightarrow P L_{\nu}$, the mass Lagrangian $\mathcal{L}$ can be decomposed into the sum of an even part and an odd part: $\mathcal{L}=\mathcal{L}^{e}+\mathcal{L}^{o}$, with $\mathcal{L}^{e} \rightarrow \mathcal{L}^{e}$ and $\mathcal{L}^{o} \rightarrow-\mathcal{L}^{o}$. This induces a decomposition of the mass matrix $M_{\nu}=M_{\nu}^{e}+M_{\nu}^{o}$, with the constraints $M_{\nu}^{e}=P M_{\nu}^{e} P$ and $M_{\nu}^{o}=-P M_{\nu}^{o} P$. Thus $M_{\nu}^{e}$ is of the form given by (4), but $M_{\nu}^{o}$ is of the form

$$
M_{\nu}^{o}=\left(\begin{array}{ccc}
0 & \nu_{12}^{\prime} & -\nu_{12}^{\prime} \\
\nu_{12}^{\prime} & \nu_{22}^{\prime} & 0 \\
-\nu_{12}^{\prime} & 0 & -\nu_{22}^{\prime}
\end{array}\right) .
$$

To calculate $\nu_{i j}^{\prime}$, we assume the atmospheric mixing angle to be maximal, and the reactor angle $\theta_{13}$ to be small but not necessarily zero. Then instead of (3), the MNS matrix to $O(\theta)$ is given by

$$
U=\frac{1}{\sqrt{2}}\left(\begin{array}{ccc}
\sqrt{2} c & \sqrt{2} s & \theta \\
-s+c \theta^{*} & c+s \theta^{*} & 1 \\
-s+c \theta^{*} & c+s \theta^{*} & -1
\end{array}\right),
$$

where $\theta \equiv \sin \theta_{13} e^{i \delta}$ and $\delta$ is the CP phase. By calculating $M_{\nu}=U^{T} M_{\nu}^{d} U$ up to $O(\theta)$, we

obtain the even part $M_{\nu}^{e}$ to be given by (4) and (5), and the odd part $M_{\nu}^{o}$ to be given by (13), with

$$
\begin{aligned}
\nu_{12}^{\prime} & =\left(m_{3}-c^{2} m_{1}-s^{2} m_{2}\right) \theta / \sqrt{2}, \\
\nu_{22}^{\prime} & =c s\left(m_{1}-m_{2}\right) \theta .
\end{aligned}
$$


This is small because of the CHOOZ bound [11].

How does this breaking of the left-handed neutrino affect the 2-3 symmetry of the righthanded neutrino? If its 2-3 symmetry is coupled and linked to the 2-3 symmetry of the lefthanded neutrino, we expect a breaking of the right-handed symmetry as well. Since there are no data to guide us how the right-handed neutrinos behave, there are many uncertainties connected with this scenario.

If the 2-3 symmetry of the right-handed neutrino is independent and unlinked to the 2-3 symmetry of the left-handed neutrino, then there is no need to break the right-handed symmetry when the left-handed symmetry is broken. In that case, $M_{R}$ remains bound by the constraint $M_{R}=P M_{R} P$, but $M_{D}$ is bound only by the constraint $M_{D}=M_{D} P$ and not the constraint $M_{D}=P M_{D}$, as the left-handed 2-3 symmetry is broken. Nevertheless, since the second and third columns of $M_{D}$ are still identical, its determinant is still zero. The conclusion of having $m_{3}=0$ therefore remain unchanged. However, since $\theta_{13} \neq 0$, the previous estimate of $m_{\nu_{e}}$ should be multiplied by a factor $\cos \theta_{13}$, and that of $m_{e e}$ should be multiplied by a factor $\cos ^{2} \theta_{13}$.

The consequence for the leptogenesis asymmetry parameter $\epsilon_{1}$ in this scenario is discussed in Ref. [12]. $\epsilon_{1}$ is now proportional to a linear combination of $\Delta m_{\odot}^{2}$ and $|\theta|^{2}$.

In conclusion, we found that in the presence of a 2-3 symmetry for the right-handed neutrinos, the spectrum for the active neutrinos is inverted, with $m_{3}=0$. This conclusion remains valid whether the left-handed 2-3 symmetry is broken or not.

This research is supported by the Natural Sciences and Engineering Research Council of Canada.

[1] Z. Maki, M. Nakagawa, and S. Sakata, Prog. Theo. Phys. 28 (1962) 870.

[2] For a review and references to the original literature, see G. Altarelli, hep-ph/0410101.

[3] C.S. Lam, Phys. Lett. B507 (2001) 214.

[4] T. Kitabayashi and M. Yasue, Phys. Rev. D67 (2003) 015006; W. Grimus and L. Lavoura, Phys. Lett. B572 (2003) 189; J. Phys. G30 (2004) 73; hep-ph/0408123; Y. Koide, H. Nishiura, K. Matsuda, T. Kikuchi, and T. Fukuyama, Phys. Rev. D66 (2002) 093006; Y. Koide, Phys. Rev. D69 (2004) 093001; R. N. Mohapatra, SLAC Summer Inst. lecture; http://www- 
conf.slac.stanford.edu/ssi/2004; hep-ph/0408187; JHEP, 0410, 027 (2004); W. Grimus, A. S.Joshipura, S. Kaneko, L. Lavoura, H. Sawanaka, M. Tanimoto, hep-ph/0408123; X.-G. He and A. Zee, Phys. Lett. B560 (2003) 87.

[5] R.N. Mohapatra and S. Nasri, Phys. Rev. D71 (2005) 033001.

[6] W. Grimus and L. Lavoura, J. Phys. G30 (2004) 1073.

[7] W. Buchmuller, M. Plumacher and P. di Bari, Phys. Lett. B547 (2002) 128.

[8] U. Seljak et al., astro-ph/0407372; G.L. Fogli et al., Phys. Rev. D70 (2004) 113003.

[9] K. Eitel, Direct Neutrino Mass Experiments, in Neutrino 2004: 21st International Conference on Neutrino Physics and Astrophysics (Paris, France, 2004).

[10] H.V. Klapdor-Kleingrothaus, Eur. Phys. J. A12 (2001) 147.

[11] M. Apollonio et al., Phys. Lett. B420 (1998) 397.

[12] R.N. Mohapatra, S. Nasri, and H. Yu, hep-ph/0502026. 